\title{
The Close AGN Reference Survey (CARS)
}

\section{What is causing Mrk 1018's return to the shadows after 30 years? ${ }^{\star}$}

B. Husemann ${ }^{1, \star \star}$, T. Urrutia ${ }^{2}$, G. R. Tremblay ${ }^{3, \dagger}$, M. Krumpe ${ }^{2}$, J. Dexter ${ }^{4}$, G. Busch ${ }^{5}$, F. Combes ${ }^{6}$, S. M. Croom ${ }^{7,8}$, T. A. Davis ${ }^{9}$, A. Eckart ${ }^{5,10}$, R. E. McElroy ${ }^{7,8}$, M. Perez-Torres ${ }^{11, \$}$, M. Powell ${ }^{3}$, and J. Scharwächter ${ }^{12}$

${ }^{1}$ European Southern Observatory, Karl-Schwarzschild-Str. 2, 85748 Garching b. München, Germany e-mail: bhuseman@eso.org

2 Leibniz-Institut für Astrophysik Potsdam (AIP), An der Sternwarte 16, 14482 Potsdam, Germany

3 Yale Center for Astronomy and Astrophysics, Yale University, 52 Hillhouse Ave., New Haven, CT 06511, USA

${ }^{4}$ Max-Planck-Institut für extraterrestische Physik, Giessenbachstr. 1, 85748 Garching, Germany

5 I. Physikalisches Institut, Universität zu Köln, Zülpicher Straße 77, 50937 Köln, Germany

${ }^{6}$ LERMA, Observatoire de Paris, College de France, PSL, CNRS, Sorbonne Univ., UPMC, 75014 Paris, France

7 Sydney Institute for Astronomy, School of Physics, University of Sydney, NSW 2006, Australia

8 ARC Centre of Excellence for All-sky Astrophysics (CAASTRO), Australia

9 School of Physics \& Astronomy, Cardiff University, Queens Buildings, The Parade, Cardiff, CF24 3AA, UK

10 Max-Planck-Institut für Radioastronomie, Auf dem Hügel 69, 53121 Bonn, Germany

11 Instituto de Astrofísica de Andalucía, Glorieta de las Astronomía s/n, 18008 Granada, Spain

12 Gemini Observatory, Northern Operations Center, 670 N. A'ohoku Pl., Hilo, Hawaii, 96720, USA

Received 4 July 2016 / Accepted 16 August 2016

\section{ABSTRACT}

\begin{abstract}
We recently discovered that the active galactic nucleus (AGN) of Mrk 1018 has changed optical type again after $30 \mathrm{yr}$ as a type 1 AGN. Here we combine Chandra, NuStar, Swift, Hubble Space Telescope and ground-based observations to explore the cause of this change. The $2-10 \mathrm{keV}$ flux declines by a factor of $\sim 8$ between 2010 and 2016 . We show with our X-ray observation that this is not caused by varying neutral hydrogen absorption along the line-of-sight up to the Compton-thick level. The optical-UV spectral energy distributions are well fit with a standard geometrically thin optically thick accretion disc model that seems to obey the expected $L \sim T^{4}$ relation. It confirms that a decline in accretion disc luminosity is the primary origin for the type change. We detect a new narrow-line absorber in Ly $\alpha$ blue-shifted by $\sim 700 \mathrm{~km} \mathrm{~s}^{-1}$ with respect to the systemic velocity of the galaxy. This new Ly $\alpha$ absorber could be evidence for the onset of an outflow or a companion black hole with associated gas that could be related to the accretion rate change. However, the low column density of the absorber means that it is not the direct cause for Mrk 1018's changing-look nature.
\end{abstract}

Key words. accretion, accretion disks - galaxies: nuclei - galaxies: Seyfert - galaxies: individual: Mrk 1018

\section{Introduction}

Active galactic nuclei (AGN) and some X-ray binaries and are thought to be powered by accretion of material onto a black hole $(\mathrm{BH})$. They commonly show significant variability at optical-to-X-ray wavelengths on short timescales; this can be well described by noise processes (e.g. Nandra et al. 1997; McHardy et al. 2004; Mushotzky et al. 2011). The variability timescale is expected to scale with the mass of the $\mathrm{BH}$ and is therefore longest for super-massive $\mathrm{BHs}(\mathrm{SMBH})$ and can reach up to several hundred years (e.g. McHardy et al. 2006). It is therefore difficult or even impossible to directly measure the long-term high-amplitude fluctuations of AGN over the required timescales.

Dramatic changes in the soft X-ray brightness or the strength of broad Balmer lines emitted in the broad line region

\footnotetext{
* Based on Cycle 17 DDT program (ID: 18789, PI: G. Tremblay) approved by the Chandra Director, Dr. Belinda Wilkes. Based on Cycle 23 DDT project with the NASA/ESA Hubble Space Telescope (ID: 14486, PI: B. Husemann) approved by HST Director Dr. Kenneth Sembach.

$\star \star$ ESO Fellow.

Einstein Fellow.

* Visiting scientist: Departamento de Física Teorica, Facultad de Ciencias, Universidad de Zaragoza, 50009 Zaragoza, Spain.
}

(BLR) have been reported in some AGN. Examples of such a "changing-look" AGN caused by absorbing clouds passing in front of the nucleus and/or variable reflection components are NGC 4151 (e.g. Puccetti et al. 2007, and references therein), NGC 1365 (e.g. Risaliti et al. 2009) and NGC 4051 (Guainazzi et al. 1998), but these cloud events can be more common (Markowitz et al. 2014). Prominent examples of AGN with appearing BLR are Mrk 1018 (Cohen et al. 1986), NGC 1097 (Storchi-Bergmann et al. 1993), and NGC 2617 (Shappee et al. 2014), and examples with disappearing BLR are NGC 7603 (Tohline \& Osterbrock 1976), Mrk 590 (Denney et al. 2014), SDSS J0159+0033 (LaMassa et al. 2015), and SDSS J1011+5442 (Runnoe et al. 2016). These events can either be explained by flares from tidal disruption events (TDEs) that are due to accretion of a star (e.g. Komossa \& Bade 1999; Halpern et al. 2004; Merloni et al. 2015), or intrinsic changes in the accretion disc flow depending on their light curves.

McElroy (2016, hereafter Paper I) reported the surprising discovery that Mrk $1018(z=0.035)$, which turned from a type 1.9 to a bright type 1 AGN around 1984, has changed back to a type 1.9 nucleus after about $30 \mathrm{yr}$. The optical continuum brightness dropped by an order of magnitude between 2010 and early 2016. While we discuss in Paper I that a TDE probably 


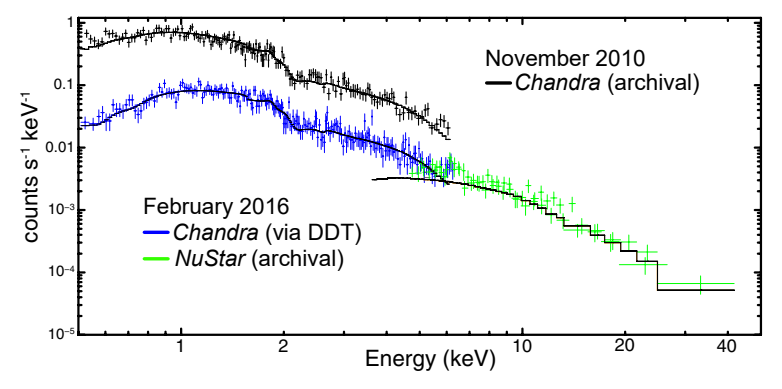

Fig. 1. Comparison of the X-ray spectrum of Mrk 1018 in November 2010 and February 2016. For all data, we show the best-fit model of a simple power law plus Galactic absorption as a solid black line. The apparent mismatch near $5 \mathrm{keV}$ between the 2016 Chandra and 2016 NuStar are instrumental effects included in the model. The X-ray flux dropped by a factor of 7.6 and both spectra are consistent with no $N_{\mathrm{H}}$ absorption.

cannot explain the variability of Mrk 1018, several other options including a cloud event still appeared possible. In this Letter, we present follow-up Director's Discretionary Time (DDT) and archival X-ray and UV spectroscopic data that show that the changing classification is driven by accretion rate changes and not by an obscuration event.

\section{Observations and results}

\subsection{Chandra and NuStar X-ray spectroscopy}

Chandra observed Mrk 1018 on 2016-02-18 as part of a DDT request. It was observed on the nominal aimpoint of the backilluminated ACIS-S3 chip for $27.2 \mathrm{ks}$. Mrk 1018 was also targeted on 2016-02-10 with NuStar (Harrison et al. 2013) for $21.6 \mathrm{ks}$ as part of the public shallow Extragalactic Survey. The combined Chandra and NuStar X-ray spectrum covering $0.5-50 \mathrm{keV}$ is shown in Fig. 1 together with an archival Chandra observation taken on 2010-09-27 (ID: 12868, PI: Mushotzky).

Both Chandra spectra were extracted with the CIAO package (v4.5) and the latest $C A L D B$ files (4.7.0) using standard settings for point sources. Similar settings for point sources were also employed for the NuStar spectral extraction using the nupipeline. Pile-up is severe only for the Chandra data from 2010 with a likelihood of $>10 \%$ in the brightest 9 pixels associated with the point spread function (PSF). To mitigate the effect of pile-up, we excluded these pixels when we extracted the spectrum and fitted a model. Afterwards we corrected for the loss of photons by fixing the model except for the normalization, which was then determined from the total spectrum in the $4-8 \mathrm{keV}$ range. All spectra were grouped with a minimum binning of 20 counts. We fitted an intrinsically absorbed power law together with absorption by the Galactic neutral hydrogen $\left(N_{\mathrm{HI}, \text { Gal }}=2.43 \times 10^{20} \mathrm{~cm}^{-2}\right.$, Kalberla et al. 2005) to the data. The Chandra and NuStar spectra from 2016 were fitted simultaneously.

The 2010 and 2016 spectra and their fits are both consistent with no absorption beyond Galactic, in particular, even partial Compton-thick absorption is ruled out with NuStar in 2016. The best-fit model parameters and errors on the photon index $(\Gamma)$ and $2-10 \mathrm{keV}$ flux are listed in Table 1 . The relative normalization of the X-ray spectra indicates that the flux has dropped by a factor of 7.6 in 2016 compared to 2010. Furthermore, there appears to be a hint of a weak $\mathrm{Fe} \mathrm{K} \alpha$ line in the 2016 data.
Table 1. X-ray observations and analysis results.

\begin{tabular}{cccccc}
\hline \hline Date $^{a}$ & $t_{\text {exp }}^{b}$ & $\theta_{\text {off }}^{c}$ & $N_{\text {bin }}\left(\chi_{v}^{2}\right)^{d}$ & $\Gamma^{e}$ & $f_{2-10 \mathrm{keV}}{ }^{f}$ \\
\hline $53587(\mathrm{~S})$ & 5.2 & 2.6 & $113(1.1)$ & $1.93 \pm 0.05$ & $1.11 \pm 0.08$ \\
$54271(\mathrm{~S})$ & 3.3 & 4.4 & $58(1.1)$ & $1.91 \pm 0.08$ & $0.92 \pm 0.10$ \\
$54273(\mathrm{~S})$ & 3.5 & 6.3 & $61(1.2)$ & $1.95 \pm 0.08$ & $0.78 \pm 0.07$ \\
$54275(\mathrm{~S})$ & 4.1 & 5.9 & $73(1.0)$ & $1.95 \pm 0.07$ & $0.85 \pm 0.07$ \\
$54628(\mathrm{~S})$ & 4.8 & 1.2 & $81(1.0)$ & $1.76 \pm 0.06$ & $0.97 \pm 0.08$ \\
$55527(\mathrm{C})$ & 22.7 & 0.0 & $169(1.2)$ & $1.68 \pm 0.04$ & $0.92 \pm 0.02$ \\
$56450(\mathrm{~S})$ & 1.3 & 2.0 & $14(1.1)$ & $1.42 \pm 0.18$ & $0.79 \pm 0.16$ \\
$56817(\mathrm{~S})$ & 2.1 & 4.5 & $3(0.5)$ & $1.50 \pm 0.60$ & $0.16 \pm 0.09$ \\
$57429(\mathrm{~S})$ & 3.7 & 3.7 & $9(1.3)$ & $1.75 \pm 0.27$ & $0.15 \pm 0.05$ \\
$57434(\mathrm{~S})$ & 3.1 & 3.9 & $8(0.5)$ & $1.33 \pm 0.26$ & $0.25 \pm 0.08$ \\
$57436(\mathrm{CN})$ & 27.2 & 0.0 & $511(1.1)$ & $1.62 \pm 0.03$ & $0.12 \pm 0.01$ \\
\hline
\end{tabular}

Notes. ${ }^{(a)}$ Modified Julian date of observations with facility indicated in brackets C-Chandra, S-Swift, N-NuStar. ${ }^{\left({ }^{b}\right)}$ Effective exposure time in ks. ${ }^{(c)}$ Off axis-angle in arcmin. ${ }^{(d)}$ Number of bins used for X-ray fitting and reduced $\chi^{2}$ of the best-fit model in brackets. ${ }^{(e)}$ Photon index with 90\% uncertainty range. ${ }^{(f)}$ Physical flux (Galactic-absorption corrected, on-axis corrected) between $2-10 \mathrm{keV}$ in units of $10^{-11} \mathrm{erg} \mathrm{cm}^{-2} \mathrm{~s}^{-1}$.

\subsection{Swift $X$-ray monitoring}

While Chandra obtained spectra with very high signal-to-noise ratio $(\mathrm{S} / \mathrm{N})$ of Mrk 1018, they only probe two epochs. The X-Ray Telescope (XRT) onboard the Swift satellite has targeted Mrk 1018 several times between 2005 and 2016 (see Table 1). With these data we infer the evolution of X-ray brightness, $N_{\mathrm{H}}$, and the $\Gamma$ based on interactive Swift data processing and analysis pipeline $^{1}$.

Given the individual exposure times of up to a few ks, only a simple spectral model consisting of a power-law plus Galactic absorption component is fitted. This simple model agrees well even with the higher $\mathrm{S} / \mathrm{N}$ Chandra observation. Pile-up does not significantly affect the Swift data because of its wider PSF and can be ignored. We list the best-fit power-law value and the 2-10 keV flux in Table 1. Considering the large uncertainties of the Swift-based quantities from February 2016, the measurements broadly agree with our DDT Chandra observation that has a much higher $\mathrm{S} / \mathrm{N}$ around the same time.

In all Swift observations with more than $2 \mathrm{ks}$, a second fit with a free Galactic absorption parameter leads to lower values or within the $90 \%$ uncertainty range of the Galactic-absorption value. Hence, during 2005 and 2016 the Swift data show no signs of additional absorption along the line of sight, in agreement with the Chandra observations. We also find some variations in $\Gamma$ ranging between $\Gamma \sim 1.9$ and $\Gamma \sim 1.6$ during the period covered by Swift observations (Fig. 2).

\subsection{HST far-UV spectroscopy}

Hubble Space Telescope (HST) FUV spectroscopy of Mrk 1018 was obtained with the Cosmic Origin Spectrograph (COS, Green et al. 2012) on February 272016 for two orbits granted as DDT. The G140L grism provides a wavelength range covering Ly $\alpha$ and C IV. A NUV acquisition image was also taken with the Primary Science Aperture and MIRRORB. The COS spectrum (Fig. 3) is compared to archival IUE spectra from 1984/86 and HST spectra taken with the Faint Object Spectrograph (FOS) in 1996.

All spectra in the bright phase exhibit a FUV continuum flux density of $(1.3 \pm 0.3) \times 10^{-14} \mathrm{erg} \mathrm{s}^{-1} \mathrm{~cm}^{-2} \AA^{-1}$ and

\footnotetext{
1 http://swift.asdc.asi.it
} 
B. Husemann et al.: What is causing Mrk 1018's return to the shadows after 30 years?

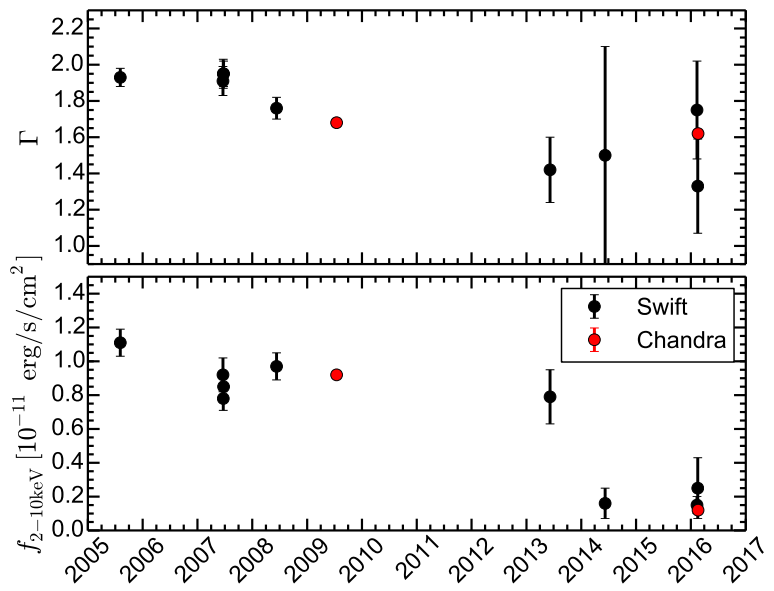

Fig. 2. Time evolution of the X-ray photon index $\Gamma$ and the $2-10 \mathrm{keV}$ flux from 2005 until 2016 based on the Swift and Chandra data.

$7.8 \times 10^{-16} \mathrm{erg} \mathrm{s}^{-1} \mathrm{~cm}^{-2} \AA^{-1}$ seen by COS, which is a factor of $\sim 17$ fainter. The Ly $\alpha$ line is shown in the lower panel of Fig. 3 after normalizing the adjacent continuum level to 1 . The broad line is well modelled with three Gaussians plus single Gaussians for each absorption line. We measure a total Ly $\alpha$ flux of $16 \times 10^{-13} \mathrm{erg} \mathrm{s}^{-1} \mathrm{~cm}^{-2}$ and $2.2 \times 10^{-13} \mathrm{erg} \mathrm{s}^{-1} \mathrm{~cm}^{-2}$, respectively, which is a factor of $\sim 7$ brighter than in 1996. The Ly $\alpha$ line width becomes narrower from $4170 \pm 62 \mathrm{~km} \mathrm{~s}^{-1}$ to $1330 \pm 122 \mathrm{~km} \mathrm{~s}^{-1}$ in FWHM. This is conceptually consistent with the resonant nature of the line. The Ly $\alpha$ photons produced in the past $30 \mathrm{yr}$ are continuously absorbed and re-emitted and thereby able to scatter to larger distance with more quiescent kinematics. This may also explain why the broad Ly $\alpha$ line appears more symmetric than the asymmetric Balmer lines, as reported in Paper I.

Three Ly $\alpha$ narrow absorption lines (NALs) can be identified in the FOS spectrum taken in 1996. Surprisingly, an additional Ly $\alpha$ NAL appears $20 \mathrm{yr}$ later. We label the NALs from 1 to 4 (Fig. 3). NAL 1 has an equivalent width (EW) of $0.65 \AA$ exactly at the systemic redshift of Mrk 1018. The new NAL 2 is blueshifted by $\sim 700 \mathrm{~km} \mathrm{~s}^{-1}$ with respect to the systemic redshift and has an EW of $0.32 \AA$. Absorbers $3+4$ are blue-shifted by more than $1500 \mathrm{~km} \mathrm{~s}^{-1}$ with EWs of $0.9 \AA$ and $0.4 \AA$ and less variable. These EWs imply $N_{\mathrm{H}}<10^{19} \mathrm{~cm}^{-2}$ and do not lead to measurable $\mathrm{X}$-ray absorption. Only absorber 1 is detected in C IV, while absorber 2 remains undetected. The lower EW of absorber 2 combined with the lower S/N at C IV during the fading phase may simply prevent a detection. Although C IV is undetected for the new NAL, the short-time variability of the line at this strength can only be produced by neutral gas within the host galaxy.

\section{Discussion}

\subsection{Inconsistency with a cloud event}

We have peculated in Paper I whether a dense cloud might be moving into our line of sight and causes the dimming of the nucleus. Such a scenario could explain the potential periodicity of such an event. For a BH mass of $M_{\mathrm{BH}} \sim 8 \times 10^{7} M_{\odot}(\mathrm{Pa}-$ per I) an orbital period of $30 \mathrm{yr}$ would correspond to a velocity of $3300 \mathrm{~km} \mathrm{~s}^{-1}$ at a mean distance of $0.03 \mathrm{pc}$. The combined highquality Chandra and NuStar spectrum clearly shows that this scenario can be reliably ruled out because no significant $N_{\mathrm{H}}$ can be detected. In particular, the high energies probed by NuStar imply that Compton-thick obscuration is excluded. The apparent

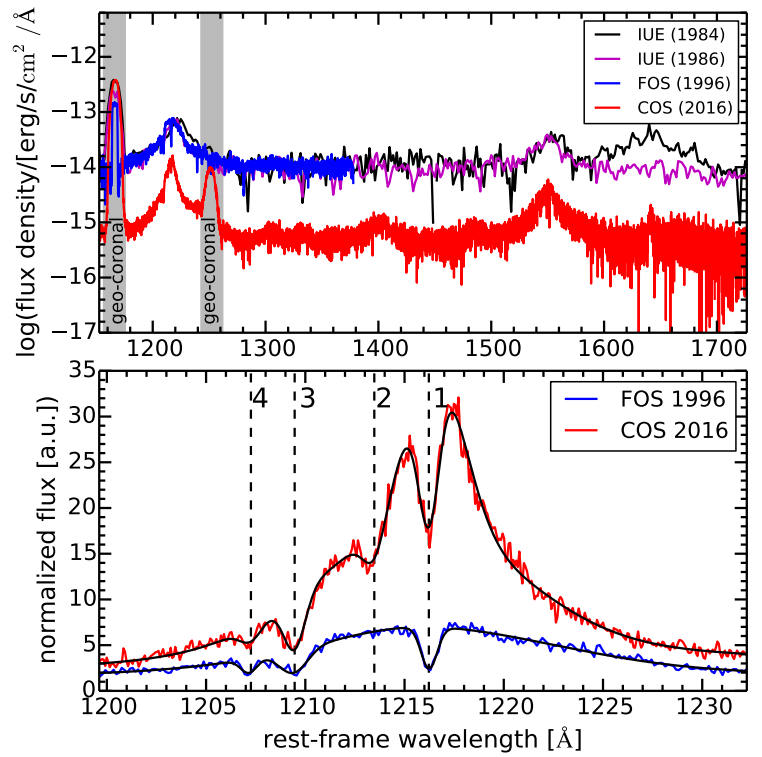

Fig. 3. Upper panel: FUV spectra of Mrk 1018 taken between 1984 and 2016 with IUE and the HST FOS and COS. Lower panel: Comparison of the $\operatorname{Ly} \alpha$ emission-line shape in 1996 and 2016. The spectra are normalized so that the adjacent continuum level is one. The solid black lines are the best-fit model as described in the text.

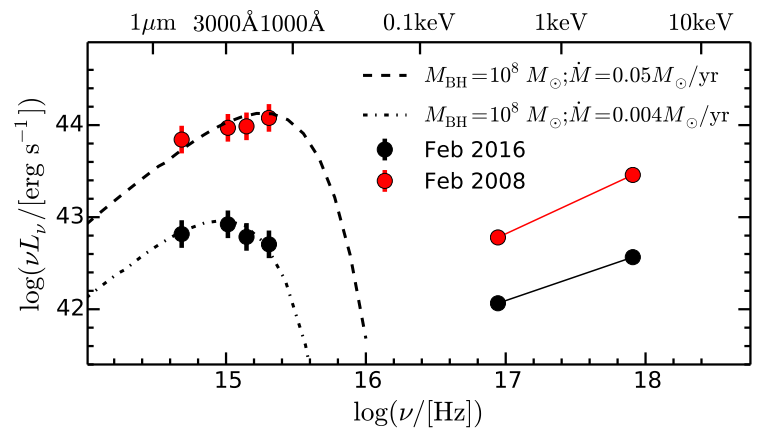

Fig. 4. Optical to X-ray SED for Mrk 1018 for two epochs. Shown are the photometry of the nucleus in the SDSS $r$ and $u$ band (see Paper I), the NUV and FUV from GALEX and HST observations, and the unabsorbed power-law X-ray spectrum from Chandra. The black dashed line represents a model for a geometrically thin relativistic accretion disc as described in the text.

time evolution in $\Gamma$ and flux without any significant $\mathrm{X}$-ray absorbing $N_{\mathrm{H}}$ column density favours a scenario in which the physical state of the accretion disc underwent a significant change or reconfiguration.

\subsection{Accretion disc changes probed by the spectral energy distribution}

GALEX observations in the NUV and FUV were taken 2008 October 21 as part of the medium imaging survey when Mrk 1018 was still in the bright phase. Within 100 days it was targeted by Swift in the X-rays and $U$ band as well as by the Palomar Transient Factory in the $r$ band. The corresponding SED of the nucleus is shown in Fig. 4. The SED significantly changes spectral shape in the optical-FUV range when Mrk 1018 was fading, as revealed by the HST observation in combination with quasisimultaneous $u$ and $r$ band photometry (see Paper I). 
We modelled the optical/UV radiation as a local black-body radiation from a geometrically thin relativistic accretion disc (Page \& Thorne 1974). Relativistic effects were included by using the GRTRANS ray tracing code (Dexter 2016). For simplicity, we assumed a Schwarzschild BH with fixed $M_{\mathrm{BH}}=10^{8} M_{\odot}$ (Paper I) and $i=15^{\circ}$ and fitted for $\dot{M}$ at each epoch. The bestfitting models with $\dot{M} \simeq 0.004$ and $0.05 M_{\odot} \mathrm{yr}^{-1}$ are shown in Fig. 4. The simple model provides a satisfactory explanation for the change in SED shape, and the inferred factor $\simeq 10$ drop in $\dot{M}$ agrees with the observed decrease in $f_{2-10 \mathrm{keV}}$.

The good agreement between a static disc model and the spectra with a difference of a factor $\simeq 10$ in luminosity implies that $L \sim T_{\text {eff }}^{4}$ (equivalent to a constant inner radius). This relation is frequently seen in BH X-ray binaries (e.g. Davis et al. 2006), but previously has not been found in samples of AGN. Their spectra typically peak at $\lambda \simeq 1000 \AA$ (Laor \& Davis 2014), close to our fit for Mrk 1018 in the bright phase $\left(\lambda_{\text {peak }} \sim 1300 \AA\right)$. The change to $\lambda_{\text {peak }} \sim 3300 \AA$, coincident with the decline in luminosity, provides evidence of an optically thick accretion disc.

Cutting the fuel supply of the accretion disc would propagate down to smaller radii on the inflow timescale, $\gtrsim 10^{2} \mathrm{yr}$ for the optical emitting region in AGN, much longer than in Mrk 1018. This timescale is consistent with the disc thermal time, however, and thermal fluctuations can explain the optical/UV variability properties in AGN accretion discs (Kelly et al. 2009). For Mrk 1018 the thermal fluctuation would have to be global, decreasing the temperature across large parts of the disc and not in small patches (Dexter \& Agol 2011).

\subsection{What is the nature of the new Ly $\alpha A L$ ?}

The most surprising result of our follow-up observations is the appearance of a new associated NAL in Ly $\alpha$ that was absent $20 \mathrm{yr}$ ago. While it appears natural that this feature is linked to the changing-look AGN event of Mrk 1018, it may also be completely unrelated. Below we discuss three potential scenarios for the origin of the NAL, but it may also be something unexpected.

Given the significant variability of the NAL strength and the radial motion of $700 \mathrm{~km} \mathrm{~s}^{-1}$ towards us, the first obvious possibility is that we see an outflow (e.g. Hamann et al. 2013). Assuming a constant outflow velocity implies a maximum distance of $0.01 \mathrm{pc}$, which is rather close to the nucleus. However, the background source may not be the accretion disc continuum, but the broad wing in Ly $\alpha$ that has larger surface area and might reach up to pc scales as a result of resonant scattering. The NAL could then originate from a much faster wind if it is slightly offset from the accretion disc along the line of sight with some inclination. The outflow scenario is attractive because it may be responsible for limiting the gas inflow by pushing gas outwards far beyond the outer disc. Disc winds seen in thermal states of BH X-ray binaries (e.g. Ponti et al. 2012) have estimated outflow rates comparable to or larger than the inflow rate. The appearing asymmetry towards the blue side of $\mathrm{H} \alpha$ and $\mathrm{H} \beta$ as reported in Paper I could be a signature of some BLR clouds that are pushed outwards, which still needs to be confirmed.

Another possibility is that the NAL gas is not associated with the accreting SMBH, but orbits a companion SMBH. This could explain the blueshift with pure gravitational motion if the approaching side of clouds around this second $\mathrm{SMBH}$ is just moving into our line of sight towards the accretion disc. Since Mrk 1018 is an advanced major merger such a binary SMBH scenario is plausible, as speculated in Paper I. The appearing line asymmetry in $\mathrm{H} \alpha$ and $\mathrm{H} \beta$ might also be signature of the gravitational interactions in a binary SMBH and not only an outflow.
Alternatively, the new NAL may be produced by a fastmoving cloud consisting of debris from the major merger. In this scenario the NAL would be disconnected from the accretion rate change. However, the speed of the cloud towards our line of sight would require a hyperbolic orbit around the SMBH.

\section{Conclusions}

Based on follow-up X-ray observations we rule out an obscuring-cloud event as the cause of the change of type again after $30 \mathrm{yr}$, as discovered in McElroy et al. (2016). All observations, in particular the optical-UV SED, are consistent with a declining accretion rate of a geometrically thin, optically thick accretion disc. Based on the appearance of a new NAL in Ly $\alpha$, we speculate whether the onset of an outflow or a putative binary SMBH system is driving instabilities in the accretion disc that cause the decline in luminosity. However, the NAL might also be completely unrelated to the accretion disc changes. Continuous monitoring from the radio to X-rays is needed to further constrain the nature of the dramatic changes at the heart of the nucleus.

Acknowledgements. We thank the anonymous referee for very constructive comments and suggestions that significantly helped to improve this Letter. GRT acknowledges support from the NASA through the Einstein Postdoctoral Fellowship Award Number PF-150128, issued by the Chandra X-ray Observatory Center, which is operated by the Smithsonian Astrophysical Observatory for and on behalf of NASA under contract NAS8-03060. MK acknowledges support by DFG grant KR 3338/3-1. MAPT acknowledges support from the Spanish MINECO through grants AYA2012-38491-C02-02 and AYA2015-63939C2-1-P. TAD acknowledges support from a Science and Technology Facilities Council Ernest Rutherford Fellowship. Parts of this research were conducted by the Australian Research Council Centre of Excellence for All-sky Astrophysics (CAASTRO), through project number CE110001020.

\section{References}

Cohen, R. D., Puetter, R. C., Rudy, R. J., Ake, T. B., \& Foltz, C. B. 1986, ApJ, 311,135

Davis, S. W., Done, C., \& Blaes, O. M. 2006, ApJ, 647, 525

Denney, K. D., De Rosa, G., Croxall, K., et al. 2014, ApJ, 796, 134

Dexter, J. 2016, MNRAS, 462, 115

Dexter, J., \& Agol, E. 2011, ApJ, 727, L24

Green, J. C., Froning, C. S., Osterman, S., et al. 2012, ApJ, 744, 60 Guainazzi, M., Nicastro, F., Fiore, F., et al. 1998, MNRAS, 301, L1 Halpern, J. P., Gezari, S., \& Komossa, S. 2004, ApJ, 604, 572

Hamann, F., Chartas, G., McGraw, S., et al. 2013, MNRAS, 435, 133 Harrison, F. A., Craig, W. W., Christensen, F. E., et al. 2013, ApJ, 770, 103 Kalberla, P. M. W., Burton, W. B., Hartmann, D., et al. 2005, A\&A, 440, 775 Kelly, B. C., Bechtold, J., \& Siemiginowska, A. 2009, ApJ, 698, 895 Komossa, S., \& Bade, N. 1999, A\&A, 343, 775

LaMassa, S. M., Cales, S., Moran, E. C., et al. 2015, ApJ, 800, 144

Laor, A., \& Davis, S. W. 2014, MNRAS, 438, 3024

Markowitz, A. G., Krumpe, M., \& Nikutta, R. 2014, MNRAS, 439, 1403

McElroy, R. E., Husemann, B., Croom, S. M., et al. 2016, A\&A, 593, L8 (Paper I)

McHardy, I. M., Papadakis, I. E., Uttley, P., Page, M. J., \& Mason, K. O. 2004, MNRAS, 348, 783

McHardy, I. M., Koerding, E., Knigge, C., Uttley, P., \& Fender, R. P. 2006, Nature, 444, 730

Merloni, A., Dwelly, T., Salvato, M., et al. 2015, MNRAS, 452, 69

Mushotzky, R. F., Edelson, R., Baumgartner, W., \& Gandhi, P. 2011, ApJ, 743, L12

Nandra, K., George, I. M., Mushotzky, R. F., Turner, T. J., \& Yaqoob, T. 1997, ApJ, 476, 70

Page, D. N., \& Thorne, K. S. 1974, ApJ, 191, 499

Ponti, G., Fender, R. P., Begelman, M. C., et al. 2012, MNRAS, 422, 11

Puccetti, S., Fiore, F., Risaliti, G., et al. 2007, MNRAS, 377, 607

Risaliti, G., Miniutti, G., Elvis, M., et al. 2009, ApJ, 696, 160

Runnoe, J. C., Cales, S., Ruan, J. J., et al. 2016, MNRAS, 455, 1691

Shappee, B. J., Prieto, J. L., Grupe, D., et al. 2014, ApJ, 788, 48

Storchi-Bergmann, T., Baldwin, J. A., \& Wilson, A. S. 1993, ApJ, 410, L11

Tohline, J. E., \& Osterbrock, D. E. 1976, ApJ, 210, L117 